\title{
Review
}

CARDIOLOGY

\section{Intravenous Sotalol: An Under Used Treatment Strategy}

\author{
Nicholas Z. Kerin \\ Wayne State University Medical School, Detroit, MI, USA
}

\author{
Keywords \\ Intravenous sotalol · Arrhythmia - Antiarrhythmic agents . \\ Treatment $\cdot$ Commentary
}

fer advantage in the treatment of AF for rate and rhythm control, as well in the pediatrics for treatment of supraventricular arrhythmias often resistant to other therapies.

(c) 2018 S. Karger AG, Basel

The pharmacologic treatment of arrhythmias has seen little advance over the past few years. Physicians treating life threatening or hemodynamically destabilizing arrhythmias depend almost entirely on intravenous (IV) amiodarone. This is regrettable due to the multiple toxicities of amiodarone and its long half-life. Once administered, it is a therapeutic commitment often to long-term therapy. Given the very long terminal elimination halflife, treatment with amiodarone may interfere with baseline electrophysiologic studies and ablation procedures. Additionally, the side effect profile can be consequential, even with brief periods of treatment. With the availability of IV sotalol, this old drug needs to be re-evaluated.

Sotalol is a racemic mixture of $\mathrm{d}$ - and $\mathrm{l}$-isomers in a 1 to 1 ratio. The d-isomer has mostly class III action, while the 1 -isomer is the beta blocker [1]. Sotalol was first introduced

Nicholas Kerin is a retired cardiologist.

\section{KARGER}

(C) 2018 S. Karger AG, Basel

E-Mail karger@karger.com

www.karger.com/crd
Nicholas Z. Kerin, MD 6076 Wildcat Run West Palm Beach, FL 33412 (USA)

E-Mail nzkerin@gmail.com 
into the U.S. in 1992 in its oral form. The drug was synthetized by AA Larcen of Mead-Johnson Pharmaceutical [2]. The drug was initially used as an anti-hypertensive and antianginal agent in Europe [3] but was found to be a potent anti-arrhythmic agent at higher doses [4]. As an anti-arrhythmic it was first introduced for ventricular arrhythmias and then later as treatment for supra-ventricular arrhythmias. Because of the oral drugs slow onset of action and long half-life (7-18 h), oral sotalol was not useful for acute emergent arrhythmias. Significant repolarization changes can be seen as early as $\mathbf{3 0}$ min with IV administration shortening the time to drug effect [5]. While the drug was widely used IV in Europe and Israel, it was not able to gain approval in the U.S. because of concern that rapid IV administration would lead to high concentrations that would cause a marked QT prolongation and Torsade de Pointes, a rapid ventricular tachycardia. It was not until Academic Pharmaceuticals developed the IV sotalol formulation employing a $5 \mathrm{~h}$ infusion did the FDA approve IV sotalol in 2009. Somberg et al. [6] reported that the pharmacokinetic parameters of IV sotalol dose of $75 \mathrm{mg}$ administered over $5 \mathrm{~h}$ was bioequivalent to an oral dose of $80 \mathrm{mg}$, and the impact on QTc prolongation was not clinically significant.

Currently, sotalol, like amiodarone, is available in both IV and oral formulations, facilitating their use in emergency situations. IV sotalol has a rapid onset of action [7] with linear pharmacokinetics [3]. In the U.S. only amiodarone and sotalol have both an IV and an oral formulation enabling transition from acute IV administration to chronic oral therapy. While sotalol's efficacy has mostly been evaluated in small clinical trials, some going back over 25 years, 2 recent meta-analysis have been informative as to the utility of sotalol.

Kerin and Jacob [8] published a meta-analysis on "The Efficacy of Sotalol in Preventing Post-Operative AF," reviewing 15 eligible publications that reviewed 20 comparisons of sotalol with an alternative. The review found sotalol to be more effective in the prevention of AF than placebo or other beta-blockers and was non-inferior to amiodarone. Initiating sotalol before cardiac surgery was no more effective than giving sotalol shortly after surgery for AF prevention. Furthermore, the IV initiation of sotalol following surgery is a more reliable method than administration via a nasogastric tube, or orally given immediately postoperatively. Both amiodarone and sotalol can cause bradycardia, hypotension, and depress LV function with both agents acting similarly. However, the incidence of clinically significant adversity with sotalol requiring cessation of therapy is infrequent. Usually reduction in dose and administering of fluids reduces these adversities.
A second systematic review by Milan et al. [9] evaluated the efficacy of sotalol in AF conversion. Ten publications were included in the meta-analysis showing sotalol superior to placebo. One noteworthy observation was the finding of AF conversion efficacy in that sotalol was not different from Class Ia and Ic anti-arrhythmic agents or amiodarone. The impression has been that amiodarone and the Ic agents were more effective than sotalol in AF conversion. Only high dose ibutilide was more effective than sotalol in AF conversion, though significantly more pro-arrhythmia was seen with ibutilide.

The issue of pro-arrhythmias has been a prime concern with sotalol and especially the IV formulation. The incidence of pro-arrhythmia reported in the product label is in the range of $3 \%$ and to be greater in patients with reduced ejection fraction. Clearly, amiodarone has a lower pro-arrhythmic risk. However, having excessive QT prolongation as a marker for potential pro-arrhythmia with sotalol is most useful and avoiding patients with EFs less than 35\% increase the safety of IV Sotalol. The general impression has been that rapid IV loading increases the risk for pro-arrhythmic toxicity, and thus, IV sotalol is to be avoided. Data contrary to this widely held belief comes from a large metaanalysis that included 37 published reports with 962 patients receiving IV sotalol [10]. Marill and Runge [10] found that "overall risk of Torsade de Pointes in patients treated with a single infusion of IV sotalol is low (0.1\%) compared with that in patients given chronic oral sotalol therapy," lower than the $2-4 \%$ pro-arrhythmic incidence seen with oral therapy. The authors employed a much faster infusion of $1.5 \mathrm{mg} / \mathrm{kg}$, or $100 \mathrm{mg}$ sotalol over $30 \mathrm{~min}$ than what is recommended in the IV sotalol product label. From this report and other case series, IV sotalol appears to have a low incidence of pro-arrhythmia when the QT is monitored and low EF patients excluded [7,11-13]. It appears that as the rate of infusion is increased, the incidence of pro-arrhythmic problems does not increase as previously thought.

An area where IV sotalol shows promise is in the treatment of pediatric arrhythmias where the toxicities associated with amiodarone may be most concerning. A retrospective review of 78 patients, $62 \%$ neonates and $46 \%$ with congenital heart disease, receiving sotalol after failing other anti-arrhythmics showed a $90 \%$ response rate to sotalol IV. These authors reported no significant QTc prolongation or pro-arrhythmia. The authors concluded that "high dose sotalol allows for a safe and rapid control of refractory tachyarrhythmias in a pediatric population" [14]. The dose of sotalol employed in this study was $152 \mathrm{mg} / \mathrm{m}^{2} /$ day (range 65-244), two-thirds greater than the median dosage of sotalol calculated by an age fac- 
tor dosing nomogram. In another case series of 83 patients, Li et al. [11] reported a $60 \%$ response rate using IV sotalol $(1 \mathrm{mg} / \mathrm{kg}$ over $10 \mathrm{~min}$ followed by a maintenance dose of $4.5 \mathrm{mg} / \mathrm{kg} /$ day) in pediatric patients with incessant tachyarrhythmias [11]. One patient had bradycardia and 2 showed significant QT prolongation requiring sotalol cessation.

Perhaps the greatest potential use of sotalol would be in the period after coronary artery bypass graft surgery to prevent postoperative AF. These patients are intensively monitored with a very high incidence of AF (30-40\%) that often prolongs a hospital stay. Gomes et al. [15] found that oral sotalol very significantly reduced postoperative AF incidence. However, the authors could not show a reduction in hospital stay (pharmacoeconomic advantage) because the time in hospital reduced by not being in AF was offset by an increased time required to load oral sotalol. IV administration would speed up the loading process and thus may well show increased efficacy in AF prevention with a pharmacoeconomic advantage (reduced hospital stay). A prospective study postoperatively using IV sotalol to prevent postoperative AF is needed to confirm what is expected, efficacy with reduced hospitalization.

To minimize the risk of sotalol caused arrhythmias, it is recommended that patients who initiated or re-initiate sotalol should be hospitalized for 3 days, or until steady state drug levels are achieved, in a facility that can provide cardiac resuscitation and continuous electrocardiographic monitoring. For this reason, patients are routinely ad- mitted for a hospital stay of 3 days. The availability of IV sotalol offers an opportunity to improve the sotalol loading protocol and decreases the length of hospital stay. According to a recent publication, administering an IV loading dose of sotalol before switching to the oral maintenance regimen can achieve steady state plasma concentration and maximum QTc prolongation within 1 day [16]. Development of a standard IV to oral dosing regimen for inpatient initiation of oral sotalol therapy to reach target maintenance dose faster may reduce hospital stay from 3 days to 1 day reducing the cost of hospitalization and the inconvenience to patients.

IV sotalol has been underutilized and with the increased experience reported in the literature, IV sotalol therapy will offer advantage in the treatment of AF for rate and rhythm control, as well in the pediatric area for the treatment of supraventricular arrhythmias often resistant to other therapies.

\section{Disclosure Statement}

The authors have no conflicts of interest to declare.

\section{Funding Sources}

The authors have no funding to declare.

\section{References}

1 Antonaccio MJ, Gomoll A: Pharmacology, pharmacodynamics and pharmacokinetics of sotalol. Am J Cardiol 1990;65:12A-21A.

2 Hara T: Cardiovascular drugs; in Petrova E (ed): Innovation in the Pharmaceutical Industry: The Process of Drug Discovery and Development. Northampton, Elgar Publishing, 2003, pp 38-61.

3 Anderson JL, Prystowsky EN: Sotalol: an important new antiarrhythmic. Am Heart J 1999; 137:388-409.

4 Anderson JL, Askins JC, Gilbert EM, Miller RH, Keefe DL, Somberg JC, Freedman RA, Haft LR, Mason JW, Lessem JN: Multicenter trial of sotalol for suppression of frequent, complex ventricular arrhythmias: a double-blind, randomized, placebo-controlled evaluation of two doses. J Am Coll Cardiol 1986;8:752-762.

5 Somberg JC, Preston RA, Ranade V, Molnar J: QT prolongation and serum sotalol concentration are highly correlated following intravenous and oral sotalol. Cardiology 2010;116:219-225.

6 Somberg JC, Preston RA, Ranade V, Molnar $\mathrm{J}$ : Developing a safe intravenous sotalol dosing regimen. Am J Ther 2010;17:365-372.
7 Ho DSW, Zecchin RP, Cooper MJ, Richards $\mathrm{DAB}$, Uther JB, Ross DL: Rapid intravenous infusion of d-1 sotalol: time to onset of effects on ventricular refractoriness, and safety. Eur Heart J 1995; 16:81-86.

8 Kerin NZ, Jacob S: The efficacy of sotalol in preventing postoperative atrial fibrillation: a meta-analysis. Am J Med 2011;124:875.e1-e9.

9 Milan DJ, Saul JP, Somberg MC, Molnar J: Efficacy of intravenous and oral sotalol in pharmacologic conversion of atrial fibrillation: a systematic review and meta-analysis. Cardiology 2017:136:52-60.

10 Marill KA, Runge T: Meta-analysis of the risk of torsades de pointes in patients treated with intravenous racemic sotalol. Acad Emerg Med 2001;8:117-124.

11 Li X, Zhang Y, Liu H, Jiang H, Ge H, Zhang Y: Efficacy of intravenous sotalol for treatment of incessant tachyarrhythmias in children. Am J Cardiol 2017;119:1366-1370.

12 Thomas SP, Guy D, Wallace E, Crampton R, Kijvanit P, Eipper V, Ross DL, Cooper MJ: Rapid loading of sotalol or amiodarone for management of recent onset symptomatic atrial fibrillation: a randomized, digoxin-controlled trial. Am Heart J 2004; 147:E3.

13 Singh SN, Tang XC, Reda D, Singh B: Systematic electrocardioversion for atrial fibrillation and role of antiarrhythmic drugs: a substudy of the SAFE-T trial. Heart Rhythm 2009;6:152-155.

14 Knudson JD, Cannon BC, Kim JJ, Moffett BS High-dose sotalol is safe and effective in neonates and infants with refractory supraventricular tachyarrhythmias. Pediatr Cardiol 2011;327:896-903.

15 Gomes JA, Ip J, Santoni-Rugiu F, Mehta D, Ergin A, Lansman S, Pe E, Newhouse TT, Chao S: Oral d,l sotalol reduces the incidence of postoperative atrial fibrillation in coronary artery bypass surgery patients: a randomized, double-blind, placebo-controlled study. J Am Coll Cardiol 1999;34:334-339.

16 Yarlagadda B, Vuddanda V, Dar T, Jazayeri MA, Parikh V, Turagam MK, Lavu M, Avula SR, Atkins D, Bommana S, Gopinathannair R, Yeruva MR, Lakkireddy D: Safety and Efficacy of Inpatient Initiation of Dofetilide versus Sotalol for atrial fibrillation. J Atr Fibrillation 2017;10:1805. 